\title{
Efeitos Perversos de Fusões Acima de Qualquer Suspeita: Um Modelo de Integração de Produtores de Bens Complementares*
}

\author{
Tarcisio Barroso da Graça ${ }^{\dagger}$
}

Sumário: 1. Introdução; 2. Revisão da Literatura; 3. O Modelo;

4. Resultados; 5. Discussão; 6. Conclu-

são.

Palavras-chave: Fusões; defesa da concorrência; bens complementares.

Códigos JEL: L40; L13.

Fusões entre firmas de bens complementares, conforme o senso comum, não causam preocupações anticoncorrenciais. Assim, as autoridades de defesa da concorrência não costumam questioná-las. Entretanto, apresentase um modelo mostrando que, em uma indústria oligopolística de bens complementares e diferenciados em que as firmas competem em preços, o sentido da mudança no nível de bem-estar do consumidor, após a integração das firmas líderes de qualidade dos respectivos bens complementares, depende da distribuição de valoração que os consumidores atribuem a tais produtos. Usa-se a família de distribuições sobre arcos elípticos, parametrizados pelo semi-eixo menor da elipse correspondente para se derivar o resultado.

According to common wisdom, mergers between complementary-goods firms should not raise anti-competitive concerns. Regulatory authorities rarely challenge them indeed. In this paper, I offer an alternative perspective in a model of price-competition in a complementary oligopoly industry with differentiated goods. The sign of the change in the consumer surplus, after the merger between the quality leaders of the respective complementary goods, depends however upon the consumers' valuation distribution over these products. A family of elliptic-arc distributions, parameterized by the elliptic small semi-axis, is used to derive the result.

\footnotetext{
*As opiniões e idéias colocadas nesse artigo não refletem, necessariamente, as das instituições às quais estou ou estive afiliado. Agradeço a Serdar Dalkir e David Eisenstadt pelas discussões, sugestões e comentários, mas assumo toda a responsabilidade por quaisquer eventuais falhas encontradas no texto.

${ }^{\dagger}$ Senado Federal. Universidade de Brasília. E-mail: tbarroso@senado.gov.br.
} 


\section{INTRODUÇÃO}

Diversos países, entre eles o Brasil, têm adotado diretrizes específicas para a investigação dos efeitos prováveis de fusões horizontais. Cowie (2001) descrevem os pormenores desse processo investigativo no caso brasileiro. Com respeito a fusões entre firmas relacionadas verticalmente, há, contudo, certa escassez de orientação prática. Em certa medida, tal escassez decorre do senso comum de que fusões verticais não provocam preocupações sobre efeitos pós-fusão anticoncorrenciais, exceto em situações particulares. Na verdade, alguns autores defendem que elas são, em geral, benéficas tanto para as firmas como para os consumidores envolvidos em virtude da eliminação da "dupla-marginalização", por exemplo.

Khemani (1999) reconhece que as fusões verticais causam, sim, preocupação sobre possíveis efeitos anticoncorrenciais, quando uma das partes ocupa uma posição dominante no seu mercado e o controle de outro mercado verticalmente relacionado pela firma dominante pode elevar as barreiras à entrada em seu mercado original. Outro fator negativo, reconhecido sob o ponto de vista da defesa da concorrência, decorre do aumento de probabilidade de ocorrência de conluio no período pós-fusão.

Nesse artigo, entretanto, apresenta-se um modelo simples cujo resultado desafia o senso comum ao mostrar que danos ao consumidor podem ocorrer por motivos distintos daqueles geralmente investigados quando se trata de fusões não-horizontais. Essa possibilidade é o elemento que gera interesse direto e imediato para as autoridades de defesa da concorrência. Afinal, de acordo com os procedimentos adotados por vários países para a análise de fusões, o tipo de integração aqui modelada não seria sequer questionado, por estar "acima de qualquer suspeita".

O modelo mostra que, em uma indústria oligopolística de bens complementares e diferenciados em que as firmas competem em preços (Bertrant competition), o sentido da mudança no nível de bem-estar do consumidor, após a integração das firmas líderes de qualidade dos respectivos bens complementares, depende da distribuição de valoração que os consumidores atribuem a tais produtos. Usa-se a família de distribuições sobre arcos elípticos, parametrizados pelo semi-eixo menor da elipse correspondente para se derivar o resultado. Conclui-se que ocorrem prejuízos aos consumidores sobre um intervalo amplo do parâmetro considerado.

A seguir, faz-se uma breve incursão sobre a literatura afeta à questão de integração de firmas ofertantes de bens complementares, evidenciando-se os principais resultados e hipóteses sob as quais estes foram obtidos. O modelo elíptico é formalmente apresentado na seção 3. Especial esforço é concentrado na derivação apropriada das funções demanda e no problema de maximização de lucros. Na seção 4, os resultados numéricos são apresentados em formato gráfico, o que permite pronta percepção de suas características qualitativas mais salientes e facilita a sua posterior discussão da seção 5 . Finalmente, a seção 6 conclui o artigo.

\section{REVISÃO DA LITERATURA}

Fusões entre fabricantes de produtos complementares não são, em geral, objeto de preocupação das autoridades de defesa da concorrência por não apresentarem características de concentração horizontal ou efeitos típicos de integração vertical, como facilitar o conluio entre os concorrentes após a concretização do negócio e/ou dificultar a entrada de novos participantes no mercado. Parte da explicação para tal condescendência parece derivar do resultado clássico de Cournot (1971) sobre o problema do monopólio complementar: preços menores dos produtos finais e aumento do bem-estar social após a fusão.

Corroborando o resultado clássico, Salinger (1989) apresenta um modelo que permite examinar o efeito de uma fusão entre firmas fabricando elementos distintos de um sistema. Assume que: (i) os elementos são usados em proporções fixas para produção do produto final, o sistema, (ii) no mercado de cada elemento, a concorrência é do tipo Cournot, isto é, na determinação das quantidades produ- 
zidas e (iii) a firma integrada comercializa apenas o pacote contendo os elementos do sistema, e não os elementos avulsos. Conclui que o equilíbrio pós-fusão caracteriza-se por maior produção e preços menores, sem que haja indício de saída de qualquer firma ou surgimento de novos óbices à entrada de novas firmas.

Ainda na mesma corrente, Economides e Salop (1992) modelam a situação em que um sistema é composto por dois elementos, cada qual produzido por duas empresas. Cada elemento é inútil fora do sistema. A estrutura do mercado de cada elemento é caracterizada como um duopólio de Cournot. Entre alternativas, os autores identificam as seguintes formas de integração das firmas: paralela vertical (similar ao caso que aqui se domina estratégia mista) e composição (similar ao caso que aqui se domina estratégia pura). Concluem que, em ambos os casos, há redução dos preços do sistema de equilíbrio no cenário pós-integração. A redução no caso "paralela vertical" é menor do que no caso "composição".

Nas palavras de Tirole (1989), "O problema da dupla marginalização (ou cadeia de monopólios) é muito similar ao do de dois produtores monopolistas de bens complementares (...) produtores monopolistas de bens complementares têm um incentivo para se integrarem com vistas a evitar a marginalização dupla e uma contração de demanda excessiva" (traduzi). Essas palavras justificam uma breve revisão sobre a literatura a respeito de monopólios e oligopólios sucessivos.

Os modelos clássicos de integração vertical, assumindo proporção fixa dos fatores, resultam em preços de equilíbrio pós-fusão menores que os iniciais, devidos à eliminação da dupla marginalização, de tal sorte que fusões assim são pró-concorrenciais. O modelo de Greenhut e Ohta (1979), em que oligopolistas sucessivos, cada qual concorrendo em seu respectivo mercado conforme Cournot, se integram verticalmente, é uma referência importante nesse contexto. Porém, Salinger (1988), mantendo as hipóteses cruciais dos modelos clássicos, alerta para o fato de que o sentido da mudança dos preços dos bens intermediários e final após a fusão depende criticamente do número de firmas não-integradas produtoras dos bens intermediários e final e do número de firmas inicialmente integradas.

A possibilidade de uso dos fatores em proporções variáveis é adicionada à análise de Greenhut e Ohta por Waterson (1982). A conclusão neste artigo depende de se a firma integrada recusa ou não suprir seus produtos para as outras firmas a jusante na cadeia produtiva. Ocorrendo a integração vertical, em caso de recusa, há aumento do bem-estar social total para elasticidades de substituição suficientemente baixas, embora os preços aumentem e seja bastante provável que o excedente do consumidor diminua. Porém, quando a recusa não é exercida ou permitida, o preço do produto final é menor e o excedente do consumidor aumenta após a concretização da integração vertical.

Em outra vertente, integrações verticais para as quais existe a possibilidade de substituição entre os insumos dos monopolistas e alguns insumos disponibilizados em mercados em concorrência saudável podem conduzir ao aumento de preços do produto final e diminuir o excedente do consumidor, ou seja, podem gerar efeitos anticoncorrenciais. Estes modelos são caracterizados por tecnologia de proporções variáveis dos insumos para a produção do produto final. Referências nessa direção são Hay (1973), Warren-Boulton (1974) e Schmalensee (1973).

Outra ramificação da literatura econômica aplicável ao presente estudo refere-se à questão estratégica sobre a formação de pacotes (bundling). Afinal, após a ocorrência da fusão entre fabricantes de bens complementares, a nova entidade adquire opções estratégicas sobre precificação que não estavam disponíveis quando as firmas eram autônomas. Entre tais opções está a comercialização de pacotes, isto é, novos produtos feitos a partir da combinação dos produtos produzidos pelas firmas originalmente. Além disso, a firma integrada pode decidir por comercializar: (i) apenas os pacotes recém-formados (estratégia pura = pure bundling), (ii) os pacotes e, também, os seus elementos constituintes separadamente (estratégia mista = mixed bundling), ou (iii) apenas os elementos, sem a formação de pacotes. Adams e Yellen (1976) estudam qual dessas três opções é mais vantajosa para um monopolista com respeito à comercialização de seus dois componentes básicos. Assumindo que os consumidores valorizam cada um dos elementos de forma independente, os autores mostram que a decisão do monopolista depende da efetiva forma de distribuição das valorações dos consumidores. Schmalensee (1984) analisa o problema quando essa distribuição é uma distribuição normal bivariada e conclui que alguns tipos 
de pacotes são preferíveis, se não houver correlação positiva perfeita entre as valorações dos consumidores. McAfee et alii (1989) adotam uma distribuição bivariada contínua e caracterizam situações em que a opção pela estratégia mista é superior às vendas avulsas. De forma geral, apesar de não haver complementaridade nesses modelos, admiti-la redundaria em consumo do pacote ou de nada no caso de monopólio sobre cada elemento. A capacidade de formação de pacotes não redunda em benefícios quando o mercado de cada elemento se caracteriza por concorrência perfeita.

\section{O MODELO}

Diferentemente dos trabalhos sobre bundling acima citados, ver-se-á que, no presente modelo, os mercados de cada um dos bens complementares são caracterizados pela competição oligopolista com produtos diferenciados. Além disso, a capacidade da firma integrada de comercializar pacotes é crucial para a derivação dos resultados aqui relatados.

Considere um sistema que pode funcionar quando seus dois elementos sejam colocados juntos (elementos A e B). Quer dizer, o sistema não é útil na ausência de um de seus elementos. O sistema é formado por uma unidade de cada elemento, i.e., é um sistema de proporção fixa 1-para-1. Cada elemento está disponível em dois níveis de qualidade: alto (H) e baixo (L).

Com relação à estrutura de mercado, apenas uma firma produz o elemento A de qualidade alta e apenas uma outra firma produz o elemento B de qualidade superior. Estas firmas não fabricam elementos de qualidade baixa. Quanto a ambos os elementos de qualidade inferior, duas ou mais firmas os manufaturam. As firmas não são integradas, isto é, cada firma produz somente um elemento. As variáveis cruciais da concorrência nessa indústria são os preços, caracterizando um jogo do tipo Bertrand em que os produtos são diferenciados.

Para cada elemento $(e \in\{A, B\})$, os consumidores $(c)$ possuem preços de reserva maiores para os tipos de qualidade superior que os preços de reserva dos respectivos elementos de qualidade menor. A diferença entre estes preços de reserva $\left(v_{e, c}\right)$ depende do elemento e do consumidor.

Com vistas à simplificação do trabalho analítico, a tecnologia é tal que não há custos fixos associados aos processos de produção dos elementos. Os custos marginais são os mesmos para todos os fabricantes dos elementos independentemente do nível de qualidade e nulos. Além disso, introduz-se uma normalização: os preços de reserva de ambos os elementos de qualidade baixa são nulos para todos os consumidores. Portanto, em função da estrutura de mercado, os preços dos elementos de baixa qualidade são zero no equilíbrio. Em consequencia, os preços dos elementos de alta qualidade assim obtidos podem ser interpretados como a diferença entre os preços dos elementos de qualidades distintas quando o equilíbrio é tal que os preços dos elementos de qualidade inferior são não-nulos. Em outras palavras, as simplificações e normalizações acima preservam o ordenamento dos resultados númericos e as conclusões qualitativas da análise.

Nesse ponto, o modelo aqui proposto assume que, para cada consumidor, o par $V=\left(v_{A, c}, v_{B, c}\right)$ pertence ao arco do primeiro quadrante de uma elipse em que o semi-eixo horizontal mede 1 unidade e o semi-eixo vertical mede $0<b \leq 1$ unidade. Outrossim, considera que os consumidores estão distribuídos contínua e uniformemente sobre o arco elíptico nordeste de tal sorte que a medida total de consumidores é normalizada e igual a 1. Generaliza, dessa forma , o modelo do arco circular $(b=1)$ de Dalkir et alii (2002).

Para o propósito do presente artigo, há três casos de interesse. O primeiro corresponde ao cenário anterior à ocorrência da fusão quando os preços dos elementos de qualidade alta são determinados de modo autônomo pelos seus respectivos fabricantes. Os outros dois casos referem-se ao período pósfusão. Uma vez integradas, as firmas líderes de qualidade podem decidir comercializar apenas o pacote contendo os elementos A e B (estratégia pura) ou oferecer ao mercado tanto o pacote como também elementos A e B avulsos (estratégia mista). Para se conduzirem as análises dos três casos, requer-se conhecer as funções demandas apropriadas. Suas derivações vêm em seguida. 


\subsection{Funções Demandas}

\subsubsection{Demanda pelos Elementos}

Nas hipóteses acima, e com o apoio visual da figura 1, deriva-se a demanda pelo elemento $A$ de qualidade superior. Quando o seu preço é $p_{A}$, a quantidade demandada é proporcional ao comprimento do arco elíptico $(V 1)$. De fato, somente os indivíduos cujos preços de reserva pelo elemento A de alta qualidade são maiores do que $p_{A}$ estão dispostos a comprá-lo a esse preço, ou seja, com valorações à direita de $p_{A}$ e à esquerda de 1 no semi eixo-horizontal positivo. Tais indivíduos são caracterizados pelo arco elíptico $(V 1)$. O fator de proporcionalidade é o comprimento do arco elíptico completo do primeiro quadrante, já que se assumiu que as valorações dos indivíduos estão uniformemente distribuídas sobre este arco completo e o tamanho da população é igual a 1. Portanto,

$$
q_{A}\left(p_{A} ; b\right)=\frac{\text { Comprimento do Arco Elíptico }(V 1)}{\text { Comprimento do Arco Elíptico }(b 1)}
$$

O ângulo $V O 1$ é tal que $\cos (V O 1)=p_{A}$, ou, invertendo, pode-se dizer que $V O 1=\arccos \left(p_{A}\right)$. O software Mathematica contém uma função interna que calcula o comprimento de arco elíptico como uma função do ângulo determinado pelos pontos extremos do arco e o centro da elipse e do quadrado da excentricidade da elipse, isto é, Comprimento do Arco Elíptico = EllipticE (ângulo central,excentricidade ${ }^{2}$ ). Para a presente derivação, em relação ao ponto $V$, o ângulo central é determinado por $\arccos \left(p_{A}\right)$ e a excentricidade é $\sqrt{1-b^{2}}$. Portanto, a função demanda pelo elemento $A$ de qualidade alta, parametrizada pelo semi-eixo menor da elipse é dada por:

$$
q_{A}\left(p_{A} ; b\right)= \begin{cases}\frac{\text { EllipticE }\left(\arccos \left(p_{A}\right) ; 1-b^{2}\right)}{\operatorname{EllipticE}\left(\frac{\pi}{2} ; 1-b^{2}\right)} & , 0 \leq p_{A} \leq 1 \\ 0 & , p_{A}>1\end{cases}
$$

De modo similar, a demanda pelo elemento B superior pode ser derivada (2). Quando o seu preço é $p_{B}$, a quantidade demandada é proporcional ao comprimento do arco elíptico $(b V)$. De fato, somente os indivíduos cujos preços de reserva pelo elemento $\mathrm{B}$ de alta qualidade são maiores do que $p_{B}$ estão dispostos a comprá-lo a esse preço, ou seja, com valorações acima de $p_{B}$ e abaixo de $b$ no semi eixo-vertical positivo. Tais indivíduos são caracterizados pelo arco elíptico $(b V)$. o fator de proporcionalidade é o comprimento do arco elíptico completo do primeiro quadrante. Portanto,

$$
\begin{aligned}
& q_{B}\left(p_{B} ; b\right)=\frac{\text { Comprimento do Arco Elíptico }(b V)}{\text { Comprimento do Arco Elíptico }(b 1)}= \\
& \qquad \frac{\text { Comprimento do Arco Elíptico }(b 1)-\text { Comprimento do Arco Elíptico }(V 1)}{\text { Comprimento do Arco Elíptico }(b 1)}
\end{aligned}
$$

Para o elemento B, o ângulo $V O 1$ é tal que $\sin (V O 1)=\frac{p_{B}}{b}$, ou, invertendo, pode-se dizer que $V O 1=\arcsin \left(\frac{p_{B}}{b}\right)$. Assim, em termos da função EllipticE:

$$
q_{B}\left(p_{B} ; b\right)= \begin{cases}\frac{\text { EllipticE }\left(\frac{\pi}{2} ; 1-b^{2}\right)-\text { EllipticE }\left(\arcsin \left(\frac{p_{B}}{b}\right) ; 1-b^{2}\right)}{\operatorname{EllipticE}\left(\frac{\pi}{2} ; 1-b^{2}\right)} & , 0 \leq p_{B} \leq b \\ 0 & , p_{B}>b\end{cases}
$$

\subsubsection{Demanda pelo Pacote}

A geometria analítica ajuda a obter a função demanda pelo pacote. Nesse caso, o arco elíptico que representa os indivíduos desejosos por adquirirem o pacote é determinado pela interseção entre o arco elíptico do primeiro quadrante e a reta que passa pelos pontos $\left(0, p_{A B}\right)$ e $\left(p_{A B}, 0\right)$, onde $p_{A B}$ é o preço do pacote. 
Para valores de $b<p_{A B} \leq 1$ (3), há apenas um ponto de interseção $(I)$ no primeiro quadrante e a sua abscissa é dada por $\frac{p_{A B}-\sqrt{b^{2}\left(1+b^{2}-p_{A B}^{2}\right)}}{1+b^{2}}$. Disso decorre que o ângulo $I O 1$ é determinado por $\arccos \left(\frac{p_{A B}-\sqrt{b^{2}\left(1+b^{2}-p_{A B}^{2}\right)}}{1+b^{2}}\right)$. Para valores de $1<p_{A B} \leq \sqrt{1+b^{2}}$ (4), há dois pontos de interseção $\left(I_{1}\right.$ e $\left.I_{2}\right)$ no primeiro quadrante. Abscissa de $\left(I_{1}\right)$ tem a mesma expressão que a de $(I)$ acima, mas a de $\left(I_{2}\right)$ é dada por $\frac{p_{A B}+\sqrt{b^{2}\left(1+b^{2}-p_{A B}^{2}\right)}}{1+b^{2}}$. Daí decorre que o ângulo $I_{2} O 1$ é determinado por $\arccos \left(\frac{p_{A B}+\sqrt{b^{2}\left(1+b^{2}-p_{A B}^{2}\right)}}{1+b^{2}}\right)$.

Assim como no caso das demandas pelos elementos, a demanda pelo pacote é expressa em termos de comprimentos de arcos elípticos:

$q_{A B}\left(p_{A B} ; b\right)= \begin{cases}1 & , 0 \leq p_{A B} \leq b \\ \frac{\text { Comprimento do Arco Elíptico }(I 1)}{\text { Comprimento do Arco Elíptico }(b 1)} & , b<p_{A B} \leq 1 \\ \frac{\text { Comp. do Arco Elíptico }\left(I_{1} 1\right)-\text { Comp. do Arco Elíptico }\left(I_{2} 1\right)}{\text { Comprimento do Arco Elíptico }(b 1)} & , 1<p_{A B} \leq \sqrt{1+b^{2}} \\ 0 & , p_{A B}>\sqrt{1+b^{2}}\end{cases}$

Em termos da função EllipticE:

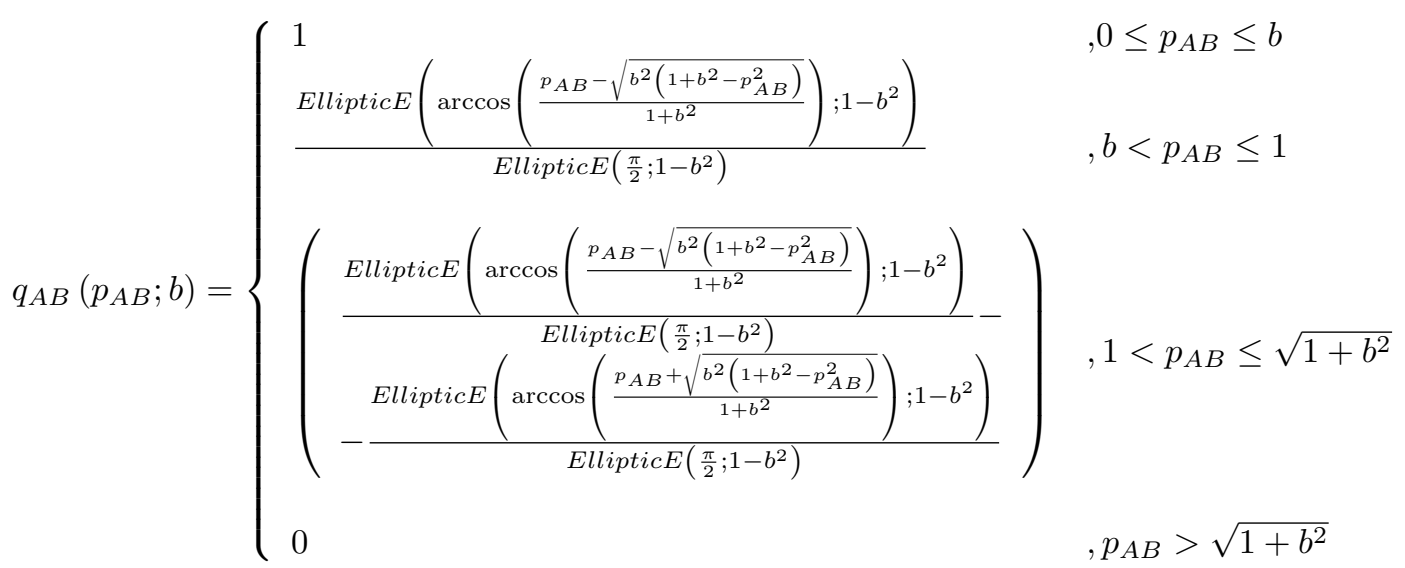

\subsection{Maximização dos Lucros}

\subsubsection{Antes da Fusão}

Antes da ocorrência da fusão, os preços dos elementos A e B, ambos de qualidade superior, são determinados de forma não-cooperativa pelos respectivos fabricantes que participam de um jogo do tipo Bertrant em que os produtos são diferenciados. Nesta fase, não existe a formação de pacote que combine os elementos A e B em um novo produto.

Nesta situação, as funções lucro dos fabricantes são dadas por:

$$
\Pi_{i}=p_{i} \times q_{i}\left(p_{i}, b\right), i=A, B
$$


Os preços $p_{i}^{*}(b), i=A, B$, são os que tornam os lucros dos fabricantes os maiores possíveis. Tais preços ótimos dependem da "forma" da elipse que caracteriza a distribuição dos preços de reserva dos consumidores com relação aos elementos de qualidade superior A e B.

Uma vez determinados os preços ótimos, podem-se determinar os lucros máximos obtidos pelos fabricantes, o excedente dos consumidores e o bem-estar social alcançados na condição de otimização dos lucros:

$$
\begin{aligned}
\Pi_{i}^{*}(b) & =p_{i}^{*}(b) \times q_{i}\left(p_{i}^{*}(b), b\right), i=A, B \\
C S^{*}(b) & =\sum_{i=A, B}\left(\int_{p_{i} \geq p_{i}^{*}(b)} q_{i}(y, b) d y\right) \\
W(b) & =\sum_{i=A, B}\left(\Pi_{i}^{*}(b)+C S_{i}^{*}(b)\right)
\end{aligned}
$$

\subsubsection{Depois da Fusão}

Após a fusão dos fabricantes dos elementos de qualidade superior A e B, a nova entidade industrial tem a possibilidade de combinar os elementos e vendê-los em um pacote como um produto novo, distinto de A e B, ampliando suas opções em termos de modos de precificação de seus produtos. Assim, a entidade recém-criada pode colocar no mercado apenas a pacote $A B$; a pacote $A B$ e os dois elementos; a pacote $\mathrm{AB}$ e apenas um dos elementos; apenas os dois elementos; ou apenas um dos elementos. Comportando-se como maximizadora de lucro, é fácil notar que a nova firma adotará uma solução que inclui a colocação no mercado do pacote $A B$. Considerar-se-ão, então, os casos em que a firma decide pelo pacote $A B$ apenas (estratégia pura), e a situação em que, além do pacote $A B$, pelo menos, o elemento B (por ser aquele que atinge o maior preço de reserva) é comercializado (estratégia mista).

\section{Estratégia Pura}

A função lucro da nova entidade é dada por: $\Pi_{A B}=p_{A B} \times q_{A B}\left(p_{A B}, b\right)$, onde $p_{A B}$ é o preço do pacote $A B$ contendo elemento de qualidade mais alta. A firma fundida maximiza seu lucro quando escolhe o preço do pacote puro $p_{A B}^{*}(b)$, que depende da parametrização da elipse sobre a qual estão distribuídas as valorações dos elementos de qualidade mais alta dos consumidores. Conhecido este preço ótimo, determinam-se o lucro máximo da firma, o excedente dos consumidores e o bem-estar social, conforme as expressões a seguir.

$$
\begin{aligned}
\Pi_{A B}^{*}(b) & =p_{A B}^{*}(b) \times q_{A B}\left(p_{A B}^{*}(b), b\right) \\
C S_{A B}^{*}(b) & =\int_{p_{A B} \geq p_{A B}^{*}(b)} q_{A B}(y, b) d y \\
W_{A B}^{*}(b) & =\Pi_{A B}^{*}(b)+C S_{A B}^{*}(b)
\end{aligned}
$$

\section{Estratégia Mista}

Neste caso, a função lucro da firma fundida é:

$$
\Pi_{A, B, A B}=p_{A} q_{A}\left(p_{A}, b\right)+p_{B} q_{B}\left(p_{B}, b\right)+p_{A B} q_{A B}\left(p_{A B}, b\right)
$$

O problema de maximização do lucro é um pouco mais complexo do que nos casos anteriores e merece melhor detalhamento:

$\operatorname{Max} p_{A} q_{A}\left(p_{A}, b\right)+p_{B} q_{B}\left(p_{B}, b\right)+p_{A B} q_{A B}\left(p_{A B}, b\right)$ 
sujeito a:

$$
\begin{aligned}
p_{A} & \geq 0 \\
p_{B} & \geq 0 \\
p_{A B} & \geq 0 \\
p_{A} & =\frac{p_{A B} b^{2}+\sqrt{b^{2}\left(1+b^{2}-p_{A B}^{2}\right)}}{1+b^{2}} \\
p_{B} & =\frac{p_{A B}+\sqrt{b^{2}\left(1+b^{2}-p_{A B}^{2}\right)}}{1+b^{2}}
\end{aligned}
$$

As duas últimas condições do problema de maximização resultam do fato de que, na situação de estratégia mista e custo marginal nulo, a nova firma escolherá preços do pacote e dos elementos de tal forma a atrair todos os consumidores e dar-lhes uma e apenas uma opção de escolha em função de seus preços de reserva ${ }^{1}$. Caso o custo marginal fosse positivo, ainda assim, haveria um relacionamento entre os preços dos elementos e o preço do pacote, que também envolveria o custo marginal, mas alguns consumidores optariam por não adquirir os produtos de qualidade superior, dados os preços ótimos. 0 processo de solução numérica do problema de maximização seria bastante mais complicado do que o do problema aqui apresentado e pouco acrescentaria, em termos qualitativos, à análise que se pretende fazer neste artigo.

Mediante estas considerações, o problema de maximização pode ser apresentado como tendo apenas uma variável de controle: o preço do pacote, $p_{A B}$. A solução que permite alcançar o maior lucro possível é $p_{A B}^{*}(b)$. Conhecida esta solução, determinam-se, pelas condições (4) e (5), os preços $p_{i}^{*}(b), i=A, B$. Daí, podem-se conhecer o lucro máximo da firma fundida, o excedente do consumidor e o bem-estar social, da seguinte forma:

$$
\begin{aligned}
\Pi_{A, B, A B}^{*}(b) & =p_{A}^{*}(b) q_{A}\left(p_{A}^{*}(b), b\right)+p_{B}^{*}(b) q_{B}\left(p_{B}^{*}(b), b\right)+p_{A B}^{*}(b) q_{A B}\left(p_{A B}^{*}(b), b\right) \\
C S_{A, B, A B}^{*}(b) & =\int_{p_{A} \geq p_{A}^{*}(b)} q_{A}(y, b) d y+\int_{p_{B} \geq p_{B}^{*}(b)} q_{B}(y, b) d y \int_{p_{A B} \geq p_{A B}^{*}(b)} q_{A B}(y, b) d y \\
W_{A, B, A B}^{*}(b) & =\Pi_{A, B, A B}^{*}(b)+C S_{A, B, A B}^{*}(b)
\end{aligned}
$$

\section{RESULTADOS}

O software Mathematica permite a determinação analítica das condições de primeira ordem dos problemas de maximização de lucros e a suas respectivas soluções numéricas, fornecido o parâmetro $b$ da elipse, assim como, perfaz os cálculos das integrais referentes aos excedentes dos consumidores ${ }^{2}$. Os cinco gráficos anexos sumarizam os resultados numéricos encontrados em função do parâmetro $b$ da elipse, que varia de 0 a 1 .

A figura 5 mostra os preços de equilíbrio das seguintes situações:

\footnotetext{
${ }^{1}$ Todos os indivíduos são atraídos a consumir, pois se houvesse algum grupo de indivíduos cujas valorações das alternativas fossem menores que os preços estabelecidos, a firma aumentaria seu lucro reduzindo um de seus preços menos que proporcionalmente ao incremento de vendas advindas da inclusão do grupo originariamente excluído. Isto se deve ao fato de que as funções-demanda dos elementos são tais que suas elasticidades-preço são superiores a 1 nas regiões próximas às extremidades do arco elíptico do primeiro quadrante. De outro lado, se houvesse um grupo de indivíduos cujas valorações fossem tais que lhes sobrasse um excedente positivo tanto se optassem pelo pacote como pelo elemento avulso aos preços estabelecidos, a firma poderia aumentar o preço do elemento, por exemplo, sem que houvesse perda de vendas globais e, conseqüentemente, aumentar seu lucro.

${ }^{2} \mathrm{O}$ autor disponibiliza a quem se interessar o programa que executa todos os cálculos cujos resultados são relatados neste artigo.
} 
- Preço do elemento A de qualidade superior antes da fusão;

- Preço do elemento B de qualidade superior antes da fusão;

- Preço do pacote $A B$ em que $A$ e $B$ são de qualidade superior, quando a firma integrada decide comercializar apenas o pacote $\mathrm{AB}$;

- Preço do pacote $A B$ em que $A$ e $B$ são de qualidade superior, quando a firma integrada decide comercializar o pacote $\mathrm{AB}$ além dos elementos avulsos;

- Preço do elemento A de qualidade superior, quando a firma integrada decide comercializar o pacote $\mathrm{AB}$ e os elementos avulsos;

- Preço do elemento B de qualidade superior, quando a firma integrada decide comercializar o pacote $\mathrm{AB}$ e os elementos avulsos.

As mesmas situações acima são aplicadas à figura 6, que apresenta as correspondentes frações de mercado de equilíbrio (pode-se falar em fração de mercado e quantidade demandada indistintamente, pois a população de consumidores tem medida 1 , por conveniência).

A figura 7 apresenta os lucros totais das firmas que produzem os elementos de qualidade superior antes e após a fusão, quando então a nova firma fundida pode comercializar sob a estratégia pura ou a estratégia mista. Analogamente, as figuras 89 apresentam os comportamentos das quantidades excedente do consumidor e bem-estar social em função do parâmetro $b$ da elipse.

\section{DISCUSSÃO}

A análise da figura 5, embora não revele supresas, proporciona alguns fatos interessantes. No período pré-fusão, os preços dos elementos $A$ e $B$ convergem à medida que a assimetria de suas respectivas valorações desaparece, isto é, $b \rightarrow 1$. Nessa convergência, o preço de $A$ é uniformemente superior ao de $B$, o que é coerente com o fato de $A$ ser o elemento que alcança preço de reserva pelo menos tão alto como o de $B$. Após a fusão, quando a firma integrada decide pela estratégia mista, ambos fenômenos também ocorrem. A diferença é que o patamar da convergência é mais elevado: o preço de $B$ antes da fusão é sempre inferior ao do mesmo elemento comercializado avulso sob a estratégia mista. o mesmo vale para $A$. Note-se que, até $b=0,53$, o preço de $A$ avulso, sob a estratégia mista, é igual a \$1, o que equivale a dizer que a firma $A$ opta por não comercializar este elemento separadamente enquanto a assimetria entre $A$ e $B$ é substancial. Ou seja, forçam-se os consumidores que valorizam bastante $A$ e pouco $B$, a levarem também $B$. Uma vez efetivada a integração, o preço do pacote sob a estratégia pura é, em todos os casos, inferior ao do sob a mista. Para $0,63<b \leq 1$, o preço daquele é constante e igual a $\$ 1$, enquanto que o deste continua a aumentar. Sob a estratégia mista, o preço do pacote pode continuar subindo além de $\$ 1$, pois os consumidores caracterizados por alto valor para $A$ e baixo valor para $B$ que deixam de comprar o pacote quando seu preço passa de $\$ 1$ são recapturados pela venda de A separadamente. Tal flexibilidade não ocorre sob a estratégia pura.

Embora não esteja explícito na figura 6, três fatos emergem da manipulação dos seus resultados: (i) a soma das frações dos consumidores que demandam $A$ e $B$ antes da fusão é maior do que $100 \%$ (ii) a fração dos consumidores que demandam o pacote sob a estratégia pura é sempre menor que $100 \%$, exceto no caso perfeitamente simétrico $(b=1)$, quando se iguala a $100 \%$; e (iii) sob a estratégia mista, a soma de tais frações de $A$, de $B$ e do pacote é igual a $100 \%$, em todos os casos. O fato (i) acontece por que, antes da fusão, as firmas líderes de qualidade de seus respectivos bens complementares maximizam seus lucros de forma não-cooperativa e, em função disso, precificam seus produtos de tal sorte que é conveniente para um determinado grupo mediano (valorações medianas de $A$ e $B$ ) de indivíduos demandar os dois produtos. Cada um dos outros dois grupos complementares extremos, dados os 
preços, escolhem demandar a apenas o elemento que lhes é mais valioso. Note-se, pois, que o grupo mediano tem a escolha de adquirir apenas $A$, apenas $B$ ou $A$ e $B$ comprados avulsos e não em pacote. Na comparação com (i), os fatos (ii) e (iii) revelam, portanto, que a fusão, qualquer que seja a estratégia de precificação, reduz as opções de escolha dos consumidores. A estratégia pura possui a característica de excluir os indivíduos que têm muito apreço por $B$ e pouco por $A$. O ritmo de inclusão aumenta bastante para $0,63<b \leq 1$, situação em que o preço ótimo do pacote estabiliza em $\$ 1$, e a quantidade demandada correspondente cresce à medida que o arco elíptico se aproxima do arco circular. Sob a estratégia mista, ao contrário, a fração do pacote diminui para $0,53<b \leq 1$, o mesmo ocorrendo com a fração de $B$. Em compensação, a fração de $A$ cresce neste intervalo, a partir de zero, de modo a compensar as reduções das frações do pacote e de $B$.

A figura 7, relativa ao total de lucros das firmas, exibe comportamento coerente com a boa intuição econômica. A soma dos lucros das firmas autônomas antes da fusão é menor do que o lucro da firma fundida em qualquer das situações de comercialização do pacote $A B$ (pura ou mista) para todos os valores de $b$. Não fosse a perspectiva de lucros maiores após a fusão, não haveria incentivo para a concretização do negócio. Além disso, o lucro auferido pela nova firma com a estratégia mista é também uniformemente superior ao obtido com a estratégia pura. Afinal, na comparação entre as estratégias, o conjunto de estratégias possíveis de precificação do caso misto contém as estratégias possíveis do caso puro.

A discussão se torna bem mais interessante quando se abordam os resultados apresentados pela figura 8, referente ao excedente do consumidor. Inicialmente, chama a atenção, a mudança de inclinação da linha relativa ao caso da estratégia pura. Para $0<b \leq 0,63$, a curva é "levemente" decrescente, mas, para $0,63<b \leq 1$ a curva é crescente. Esse comportamento se deve ao fato de que o preço ótimo do pacote puro, $p_{A B}^{*}(b)$, é tal que, para $0<b \leq 0,63$, à medida que $b$ aumenta, $p_{A B}^{*}(b)$ aumenta mais rápido do que $q_{A B}\left(p_{A B}^{*}(b), b\right)$ aumenta, pois a extremidade direita do arco elíptico está fixada no ponto $(1,0)$, "restringindo" o incremento de $q_{A B}\left(p_{A B}^{*}(b), b\right)$. A partir de $b=0,63$, porém, $q_{A B}\left(p_{A B}^{*}(b), b\right)$ aumenta ainda mais rapidamente, devido crescimento mais rápido da "convexidade" do arco elíptico da interseção, ao passo que $p_{A B}^{*}(b)$ permanece constante e igual a 1 . Deste fato, decorre a inclinação positiva do excedente do consumidor para $0,63<b \leq 1$.

Situação similar ocorre quanto ao comportamento do excedente do consumidor no caso da estratégia mista. As diferenças são: (i) o ponto de mudança da inclinação da curva é $b=0,53$ e (ii) o trecho de inclinação positiva é bem menos íngreme. A razão para esta diferença é a mesma que explica o fato de que o excedente do consumidor sob a estratégia pura é maior do que com a estratégia mista para todos os valores de $b$ : a estratégia de precificação mista confere maior flexibilidade à firma fundida para a extração do excedente do consumidor. De fato, a partir de $b=0,53$, a firma comercializando sob a estratégia mista passa a incluir em sua estratégia ótima, além dos preços do pacote e do elemento $B$, o preço do elemento A avulso. A inclusão deste grau de liberdade é responsável pela marcante separação entre o comportamento das curvas do excedente do consumidor nos casos da estratégia pura e da estratégia mista.

Finalmente, discute-se o fato mais importante que emana da figura 8. Para $b>0,92$, o excedente do consumidor após a fusão quando apenas o pacote $A B$ é comercializado é superior ao excedente do consumidor antes da fusão. Esta constatação está alinhada com os resultados clássicos. No entanto, a relação se inverte rápida e inequivocamente para $0<b \leq 0,92$, revelando a potencialidade de prejuízo ao consumidor oriundo da fusão de firmas que produzem produtos complementares. Na comparação da situação anterior à fusão e da pós-fusão com estratégia mista, o dano ao consumidor ocorre qualquer que seja a assimetria na valoração dos componentes de qualidade superior A e B, ou seja, para todos os valores de $b$.

Apesar da perda de excedente do consumidor na maior parte dos casos de distribuição elíptica de valoração dos elementos do sistema após a fusão, a figura 9 mostra que o bem-estar social total aumenta uniformemente em ambas as opções de estratégia de precificação em relação ao cenário anterior à fusão. Claramente, isto se deve ao aumento do lucro da nova entidade o qual mais que compensa 
o declínio do excedente do consumidor. Este resultado é relevante para os formuladores de política pública por explicitar o conflito que pode surgir durante a avaliação de uma proposta de fusão de fabricantes de produtos complementares. Se o critério para o julgamento for o efeito sobre o bem-estar social total, a figura 9 sugere que a aprovação deve ser concedida sem maiores questionamentos. Mas, se houver a tendência de proteção aos consumidores, e, em consequência, o excedente do consumidor for o parâmetro fundamental para a decisão, a figura 8 indica que o uso de cautela é aconselhavél. Neste caso, informações que propiciem a um melhor detalhamento de como as valorações dos consumidores atribuídas aos produtos complementares estão distribuídas ajudam a reduzir a possibilidade de uma decisão equivocada. Além disso, a figura 9 sugere que, caso seja aprovada a fusão, a vedação à comercialização do pacote e dos elementos avulsos, isto é, à estratégia mista, pode ser necessária para se evitar maior dano aos consumidores.

\section{CONCLUSÃO}

Para desafiar uma proposição considerada absolutamente verdadeira, basta oferecer um contraexemplo adequado. É o que se faz nesse artigo. A análise aqui apresentada mostra que, em um ambiente de oligopólios complementares com produtos diferenciados, uma fusão entre os fabricantes dos elementos de qualidade superior pode causar a solidificação do poder de monopólio, resultando em lucros mais altos para firma integrada ao passo que o excedente dos consumidores e suas opções de escolha são reduzidos. A especificação de uma distribuição precisa dos preços de reserva dos consumidores sobre os produtos complementares permite a determinação do equilíbrio do mercado, dos preços e dos efeitos sociais da fusão. A utilização do semi-eixo menor de elipses como parâmetro de várias distribuições resultou em uma vasta gama de estratégias de precificação no período que se segue à fusão, a maior parte das quais revela prejuízos causados aos consumidores.

Embora este estudo não derive propriedades gerais das distribuições dos preços de reserva dos consumidores, obtêm-se resultados relativamente simples que contradizem o modelo clássico de Cournot no qual a produção aumenta e os preços diminuem após a fusão de fabricantes de produtos complementares. Além disso, apontam na direção de que a forma da distribuição de valoração dos consumidores a respeito dos produtos complementares afeta o sentido da mudança do excedente do consumidor no período após a fusão.

Os resultados aqui apresentados sugerem que as atuais formas de avaliação de propostas de fusões entre fabricantes de produtos complementares de diversos países, entre eles o Brasil, pode levar as autoridades de defesa da concorrência a subestimar a possibilidade de danos anti-concorrenciais decorrentes de algumas fusões.

Se uma fusão hipotética nos moldes do modelo apresentado neste artigo fosse avaliada sob as diretrizes do guia para análise econômica de atos de concentração horizontal, tal fusão não atrairia a atenção para a possibilidade de ocorrência de danos anticoncorrenciais, pois seria fácil mostrar que as firmas proponentes do negócio atuam em mercados de produtos distintos. Do ponto de vista de efeitos não-horizontais, tampouco a integração neste formato apresenta indícios de facilitar o conluio entre concorrentes ou de dificultar a entrada no mercado de novas firmas. Destarte, é plausível que uma fusão que, como se mostrou aqui, reduza o excedente do consumidor, não seja sequer questionada pelas autoridades de defesa da concorrência. 
Figura 1 - Derivação da Função Demanda para o Elemento A

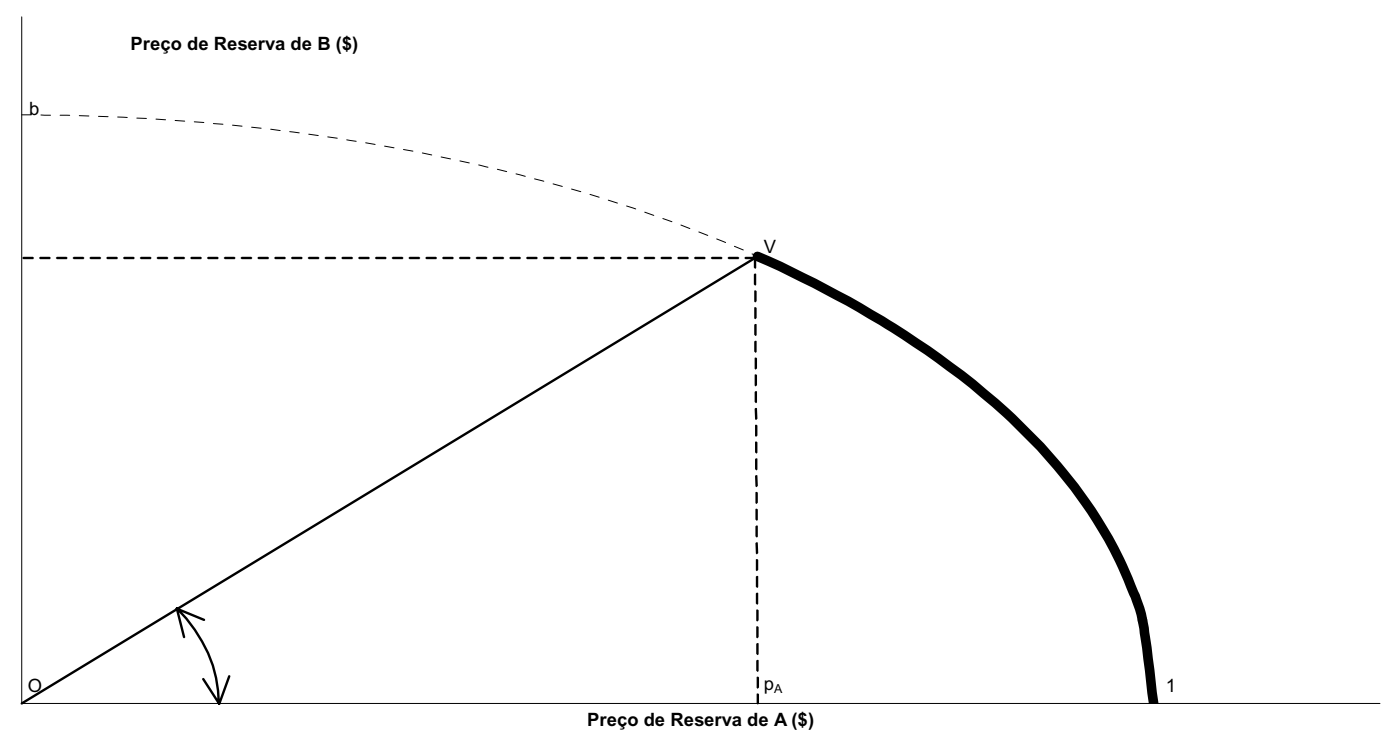

Figura 2 - Derivação da Função Demanda para o Elemento B

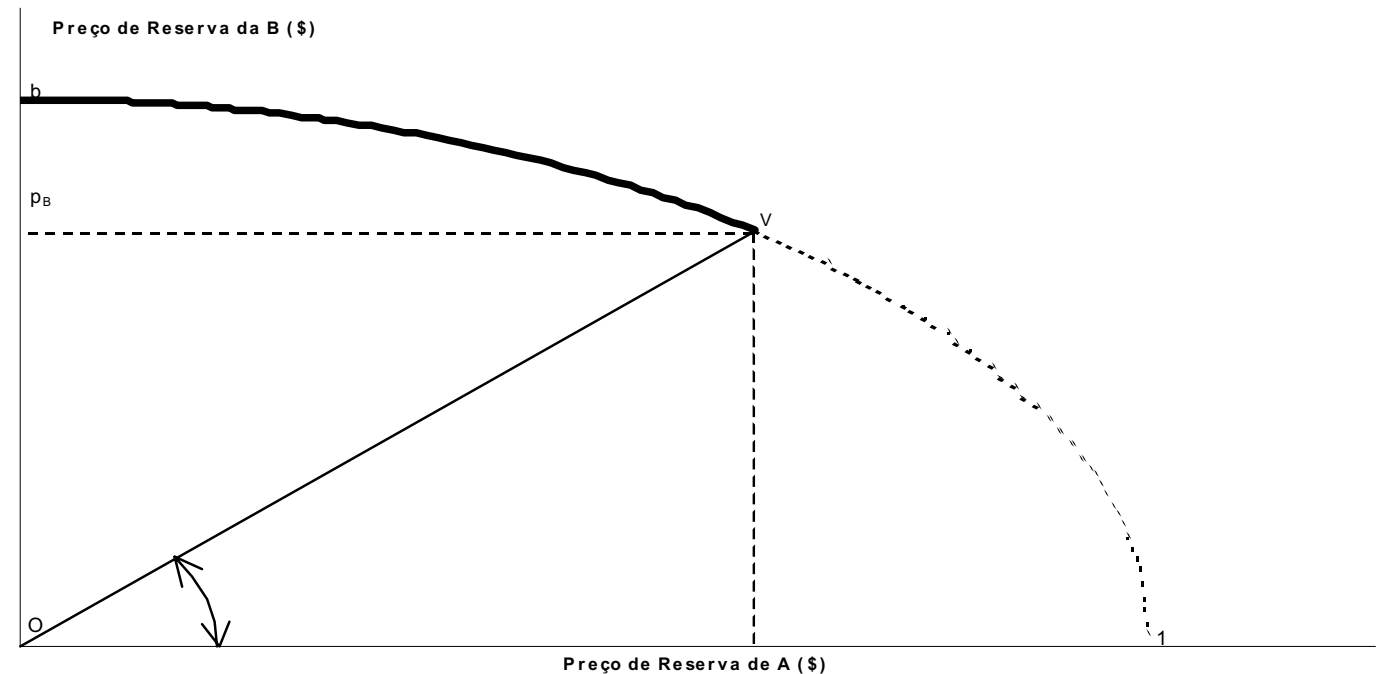


Figura 3 - Derivação da Função Demanda pelo Pacote $\mathrm{AB}$, quando $b<p A B<1$

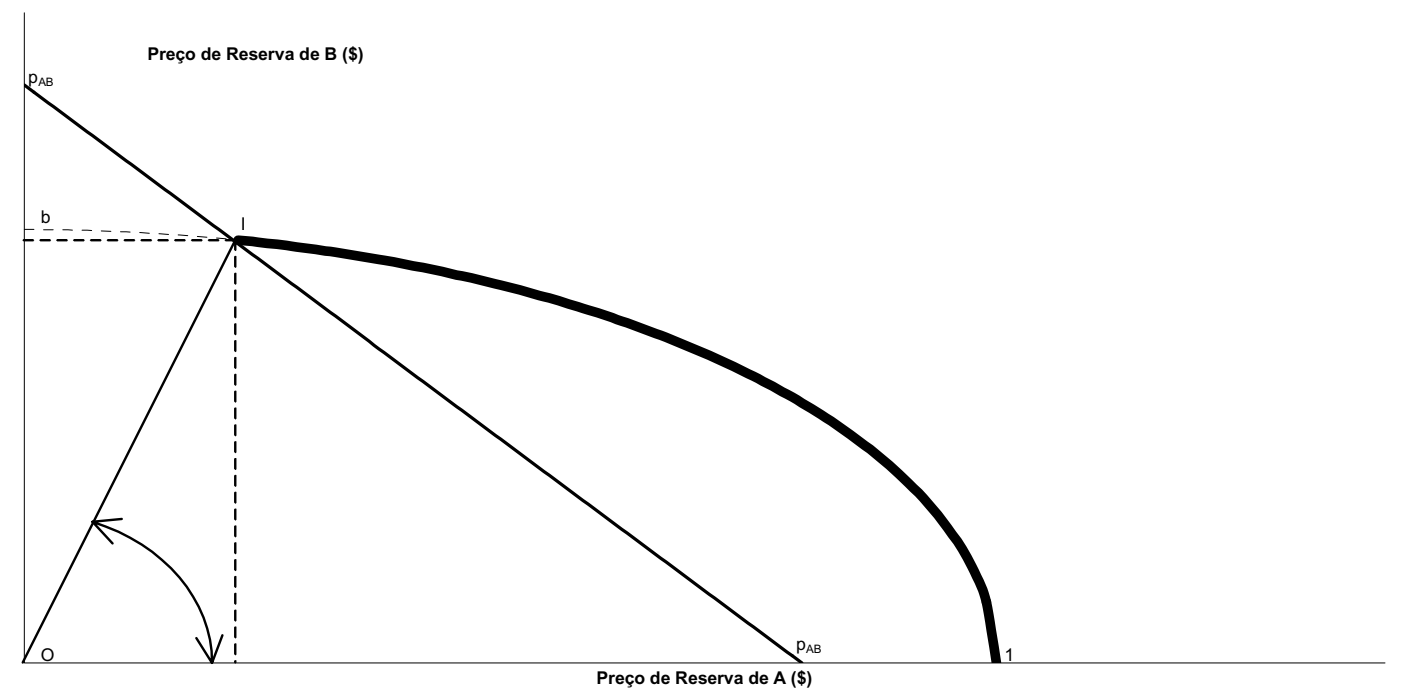

Figura 4 - Derivação da Função Demanda pelo Pacote $\mathrm{AB}$, quando $p A B>1$

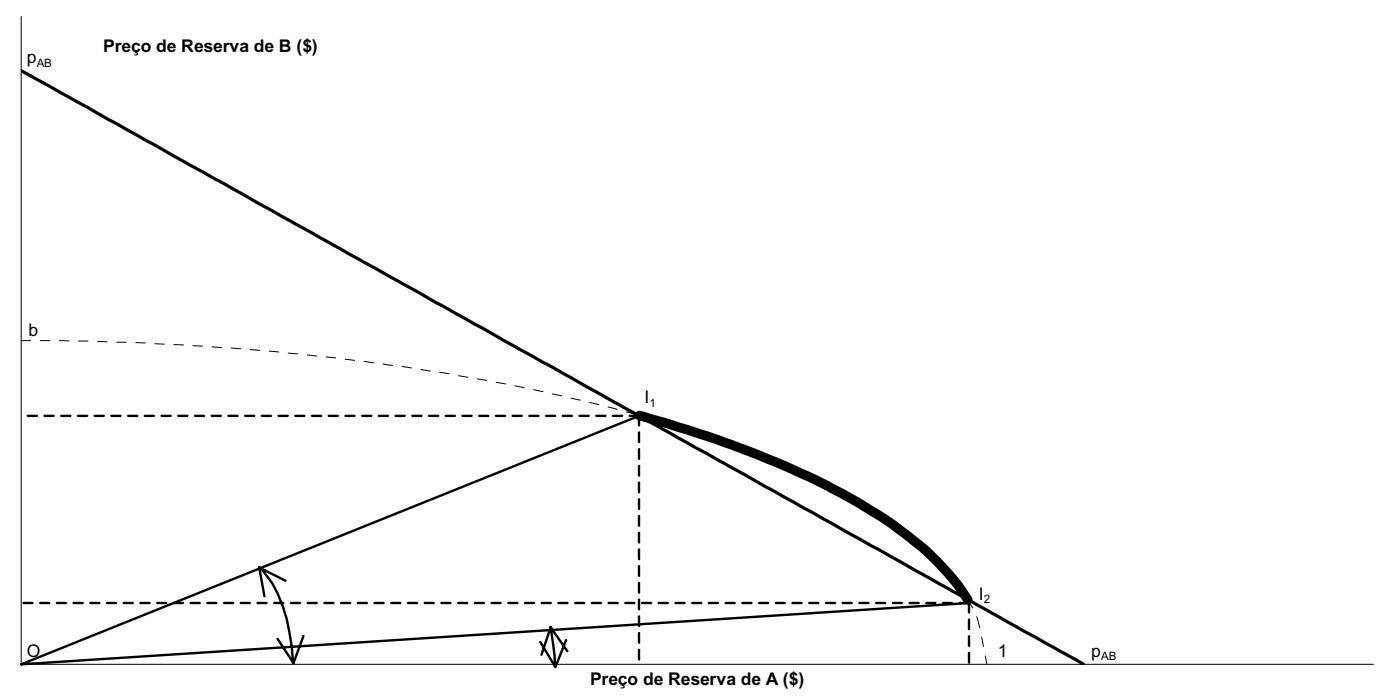


Figura 5 - Preços dos elementos A e B antes e após a fusão e dos pacotes puro e misturado

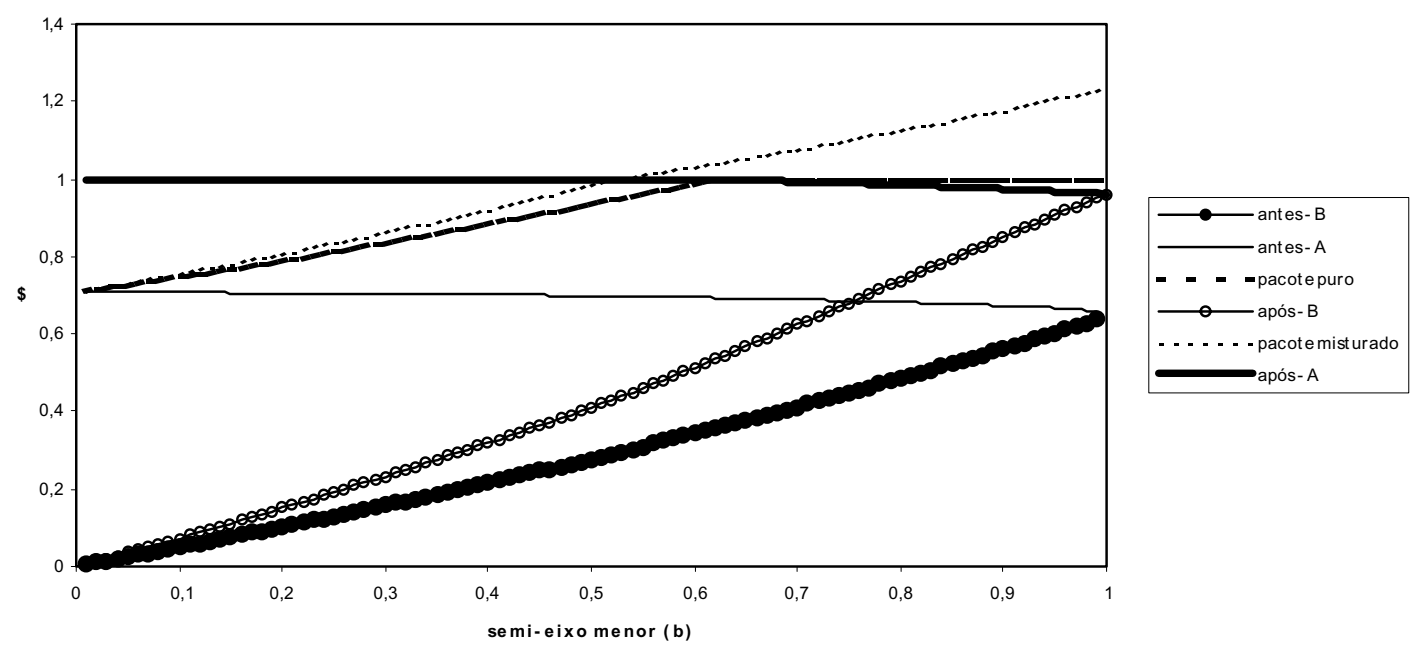

Figura 6 - Frações de mercado dos elementos A e B antes e após a fusão e dos pacotes puro e misturado após a fusão

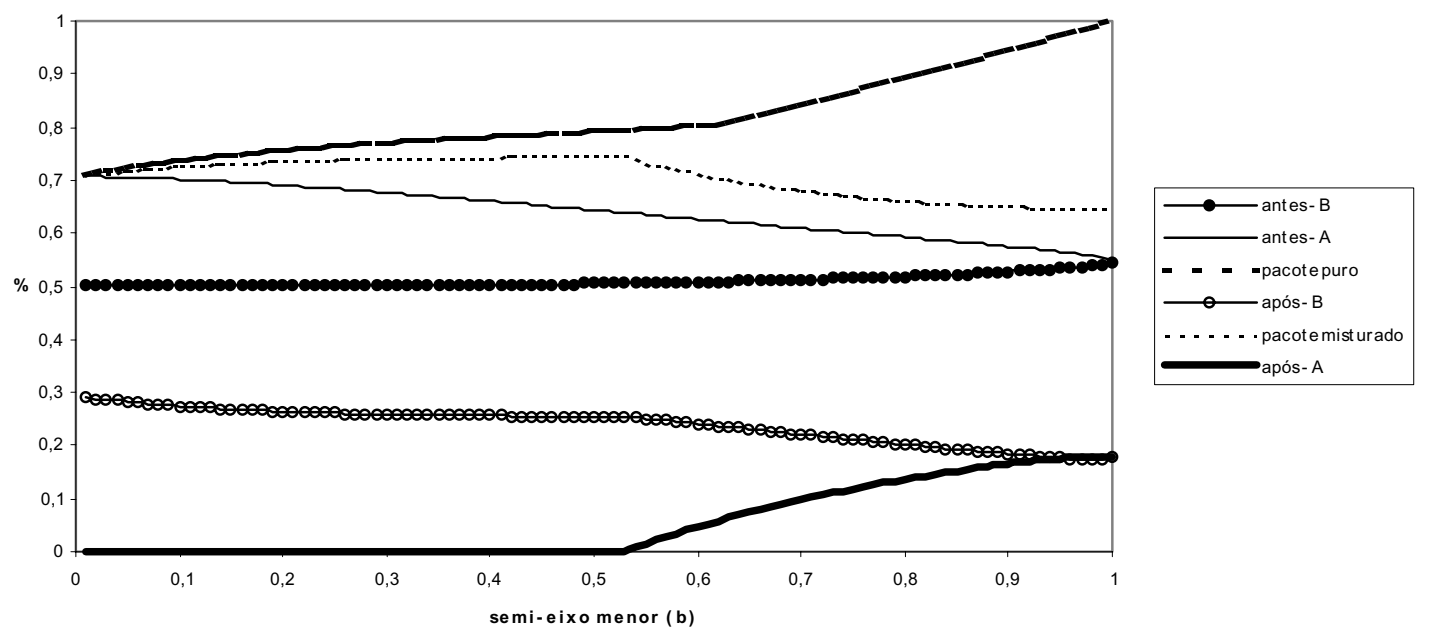


Figura 7 - Lucros antes da fusão e após a fusão com pacote puro e pacote misturado

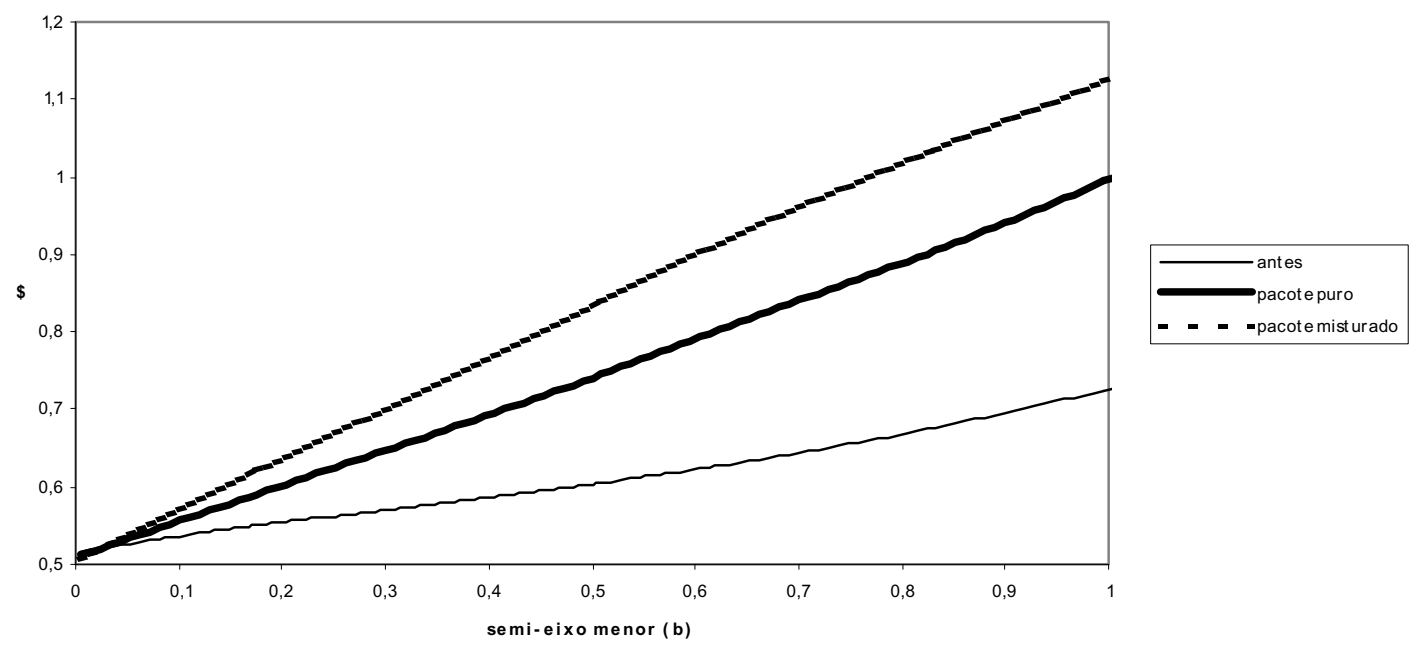

Figura 8 - Excedente do consumidor antes da fusão e após a fusão com pacote puro e com pacote misturado

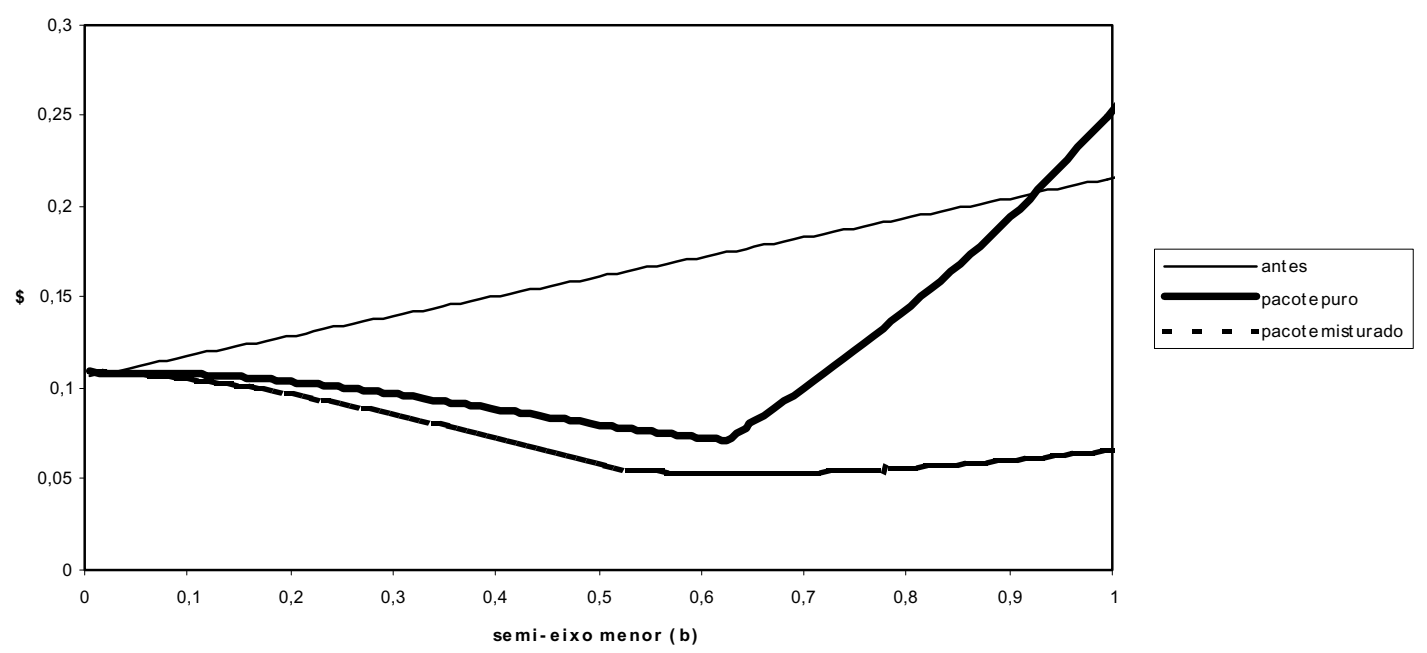


Figura 9 - Bem-estar social antes da fusão e após a fusão com pacote puro e pacote misturado

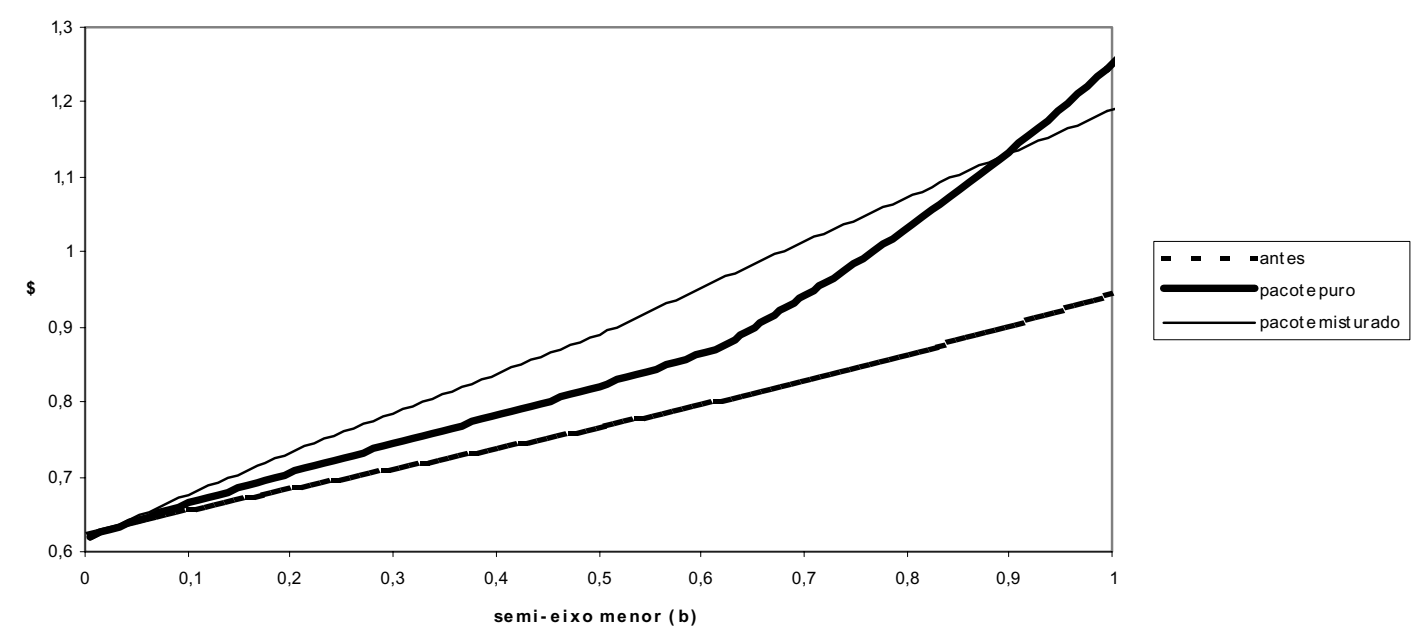




\section{Referências Bibliográficas}

Adams, W. J. \& Yellen, J. L. (1976). Commodity bundling and the burden of monopoly. The Quarterly Journal of Economics, 90(3):475-98. available at http://ideas.repec . org/a/tpr/qjecon/ v90y1976i3p475-98.html.

Cournot, A. (1971). Mathematical Principals of the Theory of Wealth, chapter IX. Augustus M. Kelley, New York.

Cowie, M., e. C. M. (2001). Antitrust review of mergers, acquisitions, and joint ventures in brazil. Revista do IBRAC, 8(3).

Dalkir, S., Eisenstadt, D., Gerstle, A., \& Masson, R. (2002). Complementary goods, monopoly vs. monopoly power: a reassessment of merger effects. Working paper, Cornell U.

Economides, N. \& Salop, S. C. (1992). Competition and integration among complements, and network market structure. Journal of Industrial Economics, 40(1):105-23. available at http: //ideas . repec . org/a/bla/jindec/v40y1992i1p105-23.html.

Greenhut, M. L. \& Ohta, H. (1979). Vertical integration of successive oligopolists. American Economic Review, 69(1):137-41. available at http://ideas.repec.org/a/aea/aecrev/ v69y1979i1p137-41.html.

Hay, G. (1973). An Economic Analysis of Vertical Integration, volume 1. Industrial Organization Review.

Khemani, R. (1999). A Framework for the Design and Implementation of Competition Law and Policy. World Bank, Washington, D.C.

McAfee, R. P., McMillan, J., \& Whinston, M. D. (1989). Multiproduct monopoly, commodity bundling, and correlation of values. The Quarterly Journal of Economics, 104(2):371-83. available at http: //ideas. repec. org/a/tpr/qjecon/v104y1989i2p371-83.html.

Salinger, M. A. (1988). Vertical mergers and market foreclosure. The Quarterly Journal of Economics, 103(2):345-56. available at http://ideas.repec.org/a/tpr/qjecon/v103y1988i2p345-56 . html.

Salinger, M. A. (1989). The meaning of "upstream"and "downstream"and the implications for modeling vertical mergers. Journal of Industrial Economics, 37(4):373-87. available at http: //ideas . repec . org/a/bla/jindec/v37y1989i4p373-87.html.

Schmalensee, R. (1973). A note on the theory of vertical integration. Journal of Political Economy, 81(2):442-49. available at http://ideas.repec.org/a/ucp/jpolec/v81y1973i2p442-49. html.

Schmalensee, R. (1984). Gaussian demand and commodity bundling. Journal of Business, 57(1):S211-30. available at http://ideas.repec.org/a/ucp/jnlbus/v57y1984i1ps211-30.html.

Tirole, J. (1989). The Theory of Industrial Organization. MIT Press.

Warren-Boulton, F. (1974). Vertical control with variable proportions. Journal of Political Economy, LXXXII.

Waterson, M. (1982). Vertical integration, variable proportions and oligopoly. Economic Journal, 92(365):129-44. available at http://ideas.repec.org/a/ecj/econjl/ v92y1982i365p129-44.html. 